\title{
Tsafon
}

Revue d'études juives du Nord

$78 \mid 2019$

L'antijudaïsme dans l'Antiquité

\section{De la rhétorique romaine à la pratique chrétienne de l'antijudaïsme : lectures, interprétations et transmission des écrits de Flavius Josèphe}

Sylvie Anne Goldberg

\section{(2) OpenEdition}

Journals

Édition électronique

URL : https://journals.openedition.org/tsafon/2247

DOI : $10.4000 /$ tsafon. 2247

ISSN : 2609-6420

Éditeur

Association Jean-Marie Delmaire

Édition imprimée

Date de publication : 1 décembre 2019

Pagination : 21-46

ISSN : $1149-6630$

\section{Référence électronique}

Sylvie Anne Goldberg, « De la rhétorique romaine à la pratique chrétienne de l'antijudaïsme : lectures, interprétations et transmission des écrits de Flavius Josèphe ", Tsafon [En ligne], 78 | 2019, mis en ligne le 01 décembre 2019, consulté le 23 juin 2021. URL : http://journals.openedition.org/tsafon/2247 ; DOI : https://doi.org/10.4000/tsafon.2247 


\title{
De la rhétorique romaine à la pratique chrétienne de l'antijudaïsme : lectures, interprétations et transmission des écrits de Flavius Josèphe
}

\author{
Sylvie Anne Goldberg*
}

\section{Rome. Premier siècle de l'ère chrétienne ${ }^{1}$}

Une génération est passée depuis la liquidation de la révolte de la Judée par les troupes menées par Titus. Quelque part, peut-être dans le Trastevere, peut-être près de la Porta Capena ou bien encore ailleurs vivent un grand nombre de Juifs. Combien? Nul ne saurait le dire avec certitude, mais apparemment beaucoup. Suffisamment en tout cas pour que leur présence soit visible. Depuis combien de temps sont-ils là ? Nul ne saurait le dire davantage, mais probablement depuis une centaine d'années. Parmi eux, un individu, peut-être étrange ou peut-être pas. Il est né, comme nombre de ses voisins, en Judée, précisément à Jérusalem vers 37 ou 38. C'est donc déjà un homme âgé qui a traversé près d'une soixantaine d'années, assisté à la fin d'un monde, s'est plié au nouveau. Il raconte qu'il est d'ascendance royale, fils de grand-prêtre, qu'il détient un savoir incommensurable, qu'il a été chef de guerre. Jadis aristocrate hiérosolymitain, désormais porte-plume. Il dit aussi qu'il a conduit ses hommes au combat, qu'il les a abandonnés à leur sort en rendant les armes car la bataille était perdue. Il se souvient de sa captivité, des chaînes qu'il portait en tant qu'esclave, se rappelle son affranchissement,

\footnotetext{
${ }^{*}$ Directrice d'études, études juives-CRH-EHESS, Paris.

${ }^{1}$ Pour cet article j'ai largement puisé dans Sylvie Anne Goldberg, «L'Amère ironie de l'histoire », Flavius Josèphe. Contre Apion, Paris, Les Belles Lettres, 2018, p. VI-XCII.
} 
l'obtention de sa citoyenneté romaine. Peut-être radote-t-il ? Il ne cesse de décrire le siège de Jérusalem, les harangues des uns et des autres, ses propres exhortations aussi, et puis le déluge de feu, l'incendie, la ruine du Temple, les monceaux de cadavres, la recherche des siens parmi les morts et les prisonniers, leur départ en déportation. À son âge il en a tant vu qu'on devrait lui pardonner de ressasser tous ces événements, mais le peut-on vraiment? Il n'est pas n'importe quel témoin innocent d'un carnage : il en est le seul narrateur !

Historien officiel de la dynastie flavienne il a été garant de sa victoire en couchant par écrit les hauts-faits qui l'ont juché sur le trône de l'Empire. Est-ce un méfait irréparable ? Est-il un traître ? Un couard opportuniste? S'agit-il d'un personnage en vue, bien en place dans la société romaine ou bien d'un simple vieillard qui a, comme on dit, « eu son heure de gloire »? Il voudrait probablement se laver de tout soupçon, se blanchir de ces accusations, mais en même temps, peut-être sent-il que ceux qui le diffament n'en ont pas qu'après lui, que c'est au fond tout son peuple qui est soumis à la vindicte. Alors il reprend le calame ou se saisit du stylet qu'il avait à peine délaissé depuis quelque temps - de nombreuses années peut-être, on ne sait! Veut-il laver les Juifs de toute charge ou bien reprendre à son compte des pamphlets qui circulent et les assaisonner à sa façon ? Ou bien encore réconforter les siens en montrant que le judaïsme, même sans temple, peut encore jouer son rôle ? Gagner la foi des «craignant Dieu »? En fait, on ne sait pas. On ne sait rien, ni de lui, ni de ce qu'il dit car il est l'unique source d'informations, tant sur sa personne que sur ce qu'il décrit; menteur ou romancier patenté, au contraire fidèle témoin, allez-donc vérifier ! Auprès de qui d'ailleurs ? Il est à peine mentionné par ses contemporains et n'apparaît en tant qu'auteur ni dans les sources hébraïques (bien plus tardives il est vrai) ni dans les romaines. On ignore même par quel nom ses proches l'appelaient: dans ses écrits, s'il se présente en tant que Joseph fils de Mathias, son nom romain était probablement Titus Flavius, tandis que la postérité n'a retenu qu'un Flavius Iosephus².

C'est pourtant l'un des écrivains ayant connu la plus large célébrité au fil des siècles, dont les œuvres, traduites dans toutes les langues, citées et récitées, lui ont fait prendre quasi valeur de Bible dans le monde

\footnotetext{
${ }^{2}$ M. Goodman, « Josephus as a Roman Citizen», dans F. Parente et J. Sievers, Josephus and the History of the Greco-Roman Period. Essays in Memory of Morton Smith, Leyde, Brill, 1994, p. 329-338. S. Mason, «Flavius Josephus in Flavian Rome : Reading On and Between the Lines ", dans A.J. Boyle et W.J. Dominik (éd.), Flavian Rome: Culture, Image, Text, Leyde-Boston, Brill, 2003, p, 559-590.
} 
chrétien. Pour un fils de grand-prêtre, même d'ascendance hasmonéenne, quel succès!

\section{Mais comment ce phénomène a-t-il été rendu possible ?}

La malice veut que ce soit probablement aux décrets édictés par Théodose (en 449) puis Justinien (536) bannissant les livres d'apparence anti-chrétienne que les écrits d'un Juif, fier de l'être, tout comme de son histoire (et bouillant de la partager avec ses contemporains), aient traversé les siècles.

Le premier ouvrage de Josèphe rapporte des événements dont les retombées ont été démesurées pour le devenir de l'Occident. La Guerre des Juifs décrit ainsi l'histoire récente (au premier siècle) de la Judée jusqu'à sa révolte contre Rome et la manière dont elle fut mâtée : le Temple incendié, la ville détruite, le tout présenté comme un châtiment divin des rebelles contre Rome. Les Antiquités judaïques, qu'il considère comme son maître ouvrage, consiste en une vaste fresque qui présente le judaïsme dans ses aspects historiques et conceptuels, depuis la création du monde selon la Bible, indiquant ses lois, ses relations aux empires et les privilèges obtenus, jusqu'à l'aube de la révolte en 66. Viennent ensuite deux ouvrages, son Autobiographie et cette apologie du judaïsme qui nous est parvenue sous le titre de Contre Apion ${ }^{3}$.

À le lire, l'étonnement tient surtout à l'appropriation chrétienne de ces œuvres. Et peu importe qu'il ait complétement ignoré ou négligé l'émergence du christianisme dont il a dû - à moins qu'elle ne soit restée totalement imperceptible pour ses contemporains - assister aux premiers frémissements ${ }^{4}$. Car son récit de la révolte de la province de Judée a servi à renforcer les fondements de la théologie chrétienne du «nouvel Israël ». Plus insolite encore, grâce à un subtil renversement et à d'habiles interprétations, son apologie du judaïsme a servi à édifier et à renforcer l'antijudaïsme. Plus simplement Josèphe, justement parce que

\footnotetext{
${ }^{3}$ L'ouvrage ne comportant aucun titre, celui-ci lui fut attribué ultérieurement. Origène et Eusèbe l'appelaient Sur l'antiquité des Juifs et Jérôme Contre Apion (Origène, Contre Celse 1.16; Eusèbe, Histoire Ecclésiastique III. 9.4-5; Préparation évangélique VIII. 7. 21 et 10.6.15).

${ }^{4}$ La diversité des courants dans le judaïsme du Second Temple était telle qu'il est probable que l'émergence en tant qu'entité distincte du christianisme dès ses débuts n'ait été qu'une construction graduelle et rétroactive ; c'est en tout cas l'hypothèse qui se dégage de certains travaux récents. Voir entre autres, P.J. Tomson et J. Schwartz (éd.), Jews and Christians in the First and Second Centuries: How to Write their History, Leyde, Brill, 2014.
} 
juif, a représenté pour les Pères de l'Église la voix sublime de l'autorité divine appuyant, si ce n'est démontrant, l'aspect paradoxal selon lequel la légitimité du christianisme, tout en se fondant sur l'authenticité d'Israël, tient à la déchéance du judaïsme. C'est par ce biais que Josèphe a été importé dans la théologie chrétienne en éminente et originelle autorité pour ce qui relevait tant de l'historicité que de la vérité des Évangiles et de la religion juive ${ }^{5}$. La chose est aisée à comprendre. Pour un chrétien, et a fortiori un chrétien non issu du judaïsme, ces ouvrages étaient une véritable aubaine. Puisque la Guerre expliquait clairement le sort funeste d'Israël, la récupération consécutive du message divin par le « véritable Israël » apparaissait logique; les Antiquités, documentation historique inégalée, permettait de mettre en scène et de visualiser les lieux des Évangiles tout en délivrant un passé riche en couleurs, en personnages et en événements. Quant au Contre Apion, formé par un curieux assemblage de deux thèmes distincts, il articulait des éléments abordés dans les Antiquités à une pure défense du judaïsme.

Défendre le judaïsme? Mais contre qui et de quoi ? Certes, l'apologie était un genre bien fréquenté par les auteurs gréco-romains ${ }^{6}$, mais à Rome, Juifs et judaïsme étaient-ils, à la fin du premier siècle, dans une situation particulièrement pénible alors que l'on estime que celle-ci ne s'est graduellement dégradée, qu'autour du $\mathrm{V}^{\mathrm{e}}$ siècle ${ }^{7}$ ? Car si la Judée était loin, que la guerre avait pris fin, que le culte juif était reconnu et, qu'en outre, le judaïsme attirait, comme jamais les gens bien nés de l'élite et de la noblesse, comment appréhender la nécessité éprouvée par Josèphe de rédiger une telle défense?

Certes, encore, on peut présumer que pour les Juifs de Rome l'ambiance était déplaisante. Les majestueuses innovations urbaines implantaient au cœur de la ville le souvenir d'une défaite qui marquait, pour eux, une fin du monde. Car la dynastie flavienne ayant établi sa légitimité sur la victoire en Judée ${ }^{8}$, elle ne cessait de la célébrer

\footnotetext{
${ }^{5}$ H. Schreckenberg, Die Flavius-Josephus-Tradition in Antike und Mittelalter, Leyde, Brill, 1972, p. 79.

${ }^{6}$ M. Edwards, M. Goodman et S. Price, avec C. Rowland (éd.), Apologetics in the Roman Empire : Pagans, Jews, and Christians, Oxford, University Press [1999], 2002.

${ }^{7}$ E.M. Smallwood, The Jews under Roman Rule. From Pompey to Diocletian, Leyde, Brill, 1976, p. 539-542. J. Juster, Les Juifs dans l'Empire romain. Leur condition économique et sociale, Paris, Geuthner, 1914, t. 2, p. 322-326.

8 J. Barclay, «Introduction », dans S. Mason (éd.), Flavius Josephus : Translation and commentary, vol. 10, Against Apion, Leyde/Boston Brill, 2007, p. XXXVI, et note 63. G. Moscati Mascetti, The «Hidden Transcripts» in Against Apion Josephus' Silent Criticism of Flavian Rome, PHD Thesis, Bar-Ilan University, 2011, p. 56-60.
} 
fastueusement. Domitien, succédant à Titus, multipliait les signes ostentatoires de la défaite judéenne par l'édification du Colisée (que l'on disait, d'ailleurs, financé par le butin de guerre) ${ }^{9}$, et des deux monumentales arches du triomphe de Titus érigées en 80-81; victoire illustrée de surcroît par l'émission de monnaies frappées du sigle Iudea capta (la Judée prise) et Iudea devicta (Judée défaite) ${ }^{10}$. Enfin, l'imposition aux Juifs de l'Empire de la taxe du fiscus judaicus remplaçant le sheqel du Temple ${ }^{11}$, conférait un goût d'amertume à la vie quotidienne des Juifs. Et, si le judaïsme fascinait quand même les Romains, le règne de Domitien (81-96) est resté marqué par la persécution des prosélytes ${ }^{12}$.

Alors contre qui Josèphe exerce-t-il son ire (s'il l'exerce vraiment) ? Sans évoquer l'arrière-plan qui, on doit l'imaginer, l'incite à reprendre la plume (d'où les difficultés de datation du texte), Josèphe recueille, dans le livre I du Contre Apion, un ensemble d'accusations et d'élucubrations colportées par des auteurs anciens sur les Juifs en des temps reculés. Si ce n'était ce florilège, personne d'ailleurs n'aurait eu connaissance de ces auteurs ni de leurs diffamations qui, restées dans la pénombre, auraient tranquillement sombré dans l'oubli. Cet opuscule constitue la source principale du corpus concernant les Juifs dans l'Antiquité. S'il est improbable de reconstituer l'ensemble des motivations qui ont inspirées Josèphe, on peut néanmoins chercher à éclairer celles qui ont conduit à sa préservation en s'intéressant à l'histoire de sa transmission au cours des siècles suivants.

\footnotetext{
${ }^{9}$ L. Feldman, « Financing the Colosseum », Biblical Archaeology Review 27, 4, JuilletAoût 2001, p. 20-31 et p. 60-61.

${ }^{10}$ L'inscription qui orne l'arche de 81 indique : «Sur l'instruction et les conseils de son père, il soumit la race des Juifs et détruisit la ville de Jérusalem qui fut attaquée en vain par tous les autres chefs, rois et peuples avant lui ou jamais ne le fut », M. Goodman, Rome et Jérusalem. Le choc des civilisations, Paris, Perrin [2009] 2011, p. 592-593. Voir la description de ces arches, dont l'une a disparu mais dont on a récemment exhumé les fondations, ainsi que les monnaies également dans la récente somme de S. Mason, A History of the Jewish War A.D. 66-70, Cambridge, University Press, 2016.

${ }^{11}$ M. Goodman, The Ruling Class of Judea : The Origins of the Jewish Revolt Against Rome A.D. 66-70, Cambridge/New York, University Press [1987] 1989, p. 236-239.

${ }^{12} \mathrm{~L}$ 'information sur les persécutions provient essentiellement du récit de Suétone, transmise ensuite par la seule patristique (Domitien 12, 2), cf. T. Reinach, Textes d'auteurs grecs et romains relatif au Judaïsme, Hildesheim/Zurich /New York, Georg Olms, [1895] 1963, p. 333. L. Feldman, Josephus' Interpretation of the Bible, Berkeley, University of California Press, 1998, p. 48. Les persécutions de Domitien ont par ailleurs été mises en doute : B.W. Jones, The Emperor Domitian, New York, Routledge, 1992. En réaction: M. Wilson, "After the Silence : Tacitus, Suetonius, Juvenal», dans A.J. Boyle and J.W. Dominik, Flavian Rome..., op. cit., 2003, p. 523-542.
} 
Transmission partielle cependant, car aucun texte grec complet du Contre Apion n'a été préservé ${ }^{13}$. Un manuscrit du $\mathrm{XI}^{\mathrm{e}}$ siècle a été transmis, amputé d'une grande part du livre II (2.52-113), qu'une version latine du $\mathrm{VI}^{\mathrm{e}}$ siècle, commandée par l'ancien sénateur romain Cassiodore ( ca 590-583) qui le fit maladroitement traduire et placer à la suite des Antiquités, permet de reconstruire ${ }^{14}$. Les raisons qui ont incité les Pères de l'Église à en omettre des passages, alors qu'ils s'étaient, semble-t-il, attachés à transmettre méticuleusement ses autres écrits, demeurent d'autant plus suspectes ${ }^{15}$ que l'absence des phrases insidieuses d'auteurs romains contemporains tels que Cicéron, Tacite, Quintilien ou Sénèque détonne dans cette anthologie ${ }^{16}$.

Une lecture superficielle du Contre Apion laisse donc penser qu'une atmosphère antijuive s'était développée à Rome. Josèphe concentre ses objections sur des citations du prêtre égyptien Manéthon (Manéthôs), remontant au VIII ${ }^{\mathrm{e}}$ siècle av. è.c. ${ }^{17}$, et du grammairien d'Alexandrie Apion. Personnage connu en son temps pour ses écrits et excentricités $^{18}$, maître de Néron, auteur d'une histoire d'Égypte et d'un glossaire homérique ${ }^{19}$, Apion aurait participé à la délégation alexandrine

\footnotetext{
${ }^{13}$ Sur la transmission du texte : H. Schreckenberg, Die Flavius-Josephus-Tradition in Antike und Mittelalter, Leyde, Brill, 1972; et id., Rezeptionsgeschichtliche und Textkritische Untersuchungen zu Flavius Josephus, Leyde, Brill, 1977. Également T. Leoni, «The Text of the Josephan Corpus : Principal Greek Manuscripts, Ancient Latin Translations, and the indirect tradition » dans H.H. Chapman et Z. Rodgers (éd.), A Companion to Josephus, Oxford, Wiley-Blackwell, 2016, p. 307-321.

${ }^{14}$ J.W. Halporn, Cassiodorus : Institutions of Divine and Secular Learning and On the Soul, Liverpool, University Press, 2003, p. 149-150. D.B. Levenson et T.R. Martin, "The Ancient Latin Translations of Josephus », dans H.H. Chapman et Z. Rodgers (éd.), A Companion..., op. cit., p. 322-344.

${ }^{15}$ L. Feldman, «Reading Between the Lines : Appreciation of Judaism in Anti-Jewish Writers Cited in Contra Apionem », dans L. Feldman et J. Levison (éd.), Josephus' Contra Apionem : Studies in its Character and Context with a Latin Concordance to the Portion Missing in Greek, Leyde, Brill, 1996, p. 250-270 ; 251.

16 Cicéron, Pro Flacco, 62-66. Tacite, Histoire 5.2.3, 5.4.4, 5.10.1, 5.13.3. Quintilien, Institutio Oratoria, III. 7,23 ; Sénèque, Traité de la superstition, frag. 41-43, transmis par St Augustin, La Cité de Dieu, VI. 11, cf Reinach, Textes..., op. cit., p. 262-264.

${ }^{17}$ Les chercheurs se sont beaucoup interrogés sur la teneur réelle de son texte, dont ils ont identifié plusieurs versions parmi les citations de Josèphe, de Jules l'Africain, et d'Eusèbe. Un point sur les récentes recherches dans J. Barclay, «Appendix 1: Manetho », dans S. Mason (éd.), Flavius Josephus ... op. cit, p. 335-337.

${ }^{18}$ P.W. van der Horst, «Who Was Apion? " dans id (éd.), Japheth in the Tents of Shem : Studies on Jewish Hellenism in Antiquity, Leuven, Peeters, 2002, p. 207-221.

${ }^{19}$ Josèphe aurait habilement monté sa critique sur la base de ces deux ouvrages, mêlant ainsi le travail de l'historien et de l'intellectuel au sens grec du terme grammatikos, selon J. Dillery, «Putting Him Back Together Again: Apion Historian, Apion Grammatikos », Classical Philology, 98, 4, Octobre 2003, p. 383-390.
} 
venue accuser les Juifs auprès des empereurs Caligula et Claude après les émeutes de 38, relatées par Philon ${ }^{20}$. De ses écrits ne sont restées que quelques bribes, dont la plupart, rapportées par Josèphe, ont servi à alimenter cette conviction.

\section{Antisémitisme : au-delà du mot, de quoi parle-t-on ?}

Depuis que l'histoire des Juifs s'est instituée en domaine indépendant au XIX ${ }^{\mathrm{e}}$ siècle, la question de l'animosité à l'égard des Juifs et de ses avatars, les persécutions qu'ils ont subies au fil des âges, l'accompagne. Mais le $\mathrm{XX}^{\mathrm{e}}$ siècle les a tant distancées dans la violence meurtrière avec la destruction des Juifs, que la question a pris un tour nouveau. Toutefois, alors que les recherches des décennies suivant la Seconde Guerre mondiale s'étaient fondées sur l'idée que cela ne pourrait désormais «plus jamais» arriver, supposant que l'on avait touché le fond de l'abîme avec les exterminations massives, les relents contemporains issus de remugles insondables ont remis l'ouvrage sur le métier.

Pour tenter de comprendre ces pulsions assassines les historiens avaient pris l'habitude de nuancer, pour mieux les distinguer, les positions théologiques chrétiennes antijuives, des attitudes sociales et/ou politiques antisémites modernes dénuées de contenu religieux et plus ancrées dans des relations d'antagonisme. En effet, depuis les travaux pionniers de James Parkes ${ }^{21}$, Joshua Trachtenberg ${ }^{22}$, Jules Isaac ${ }^{23}$, Léon Poliakov $^{24}$, Bernhard Blumenkranz ${ }^{25}$, les études se sont démultipliées afin de tenter de définir les constructions, développements et degrés d'intensité de ce rapport insolite qu'entretient l'Occident avec les Juifs, tout en tâchant de circonscrire les cadres de ce qui a fini par constituer

\footnotetext{
${ }^{20}$ Philon d'Alexandrie, Legat. 114-134; Flacc., cf P.W. van der Horst, Philo's Flaccus : The First Pogrom: Introduction, Translation, and Commentary, Leyde, Brill, 2003.

${ }^{21}$ J. Parkes, The Conflict of the Church and the Synagogue : A Study in the Origins of Anti-Semitism, Londres, Soncino Press, 1934.

22 J. Trachtenberg, The Devil and the Jews : The Medieval Conception of the Jew and its Relation to Modern Antisemitism, [1943] Philadelphie, JPS, 2002.

${ }^{23}$ J. Isaac, Genèse de l'antisémitisme. Essai historique, Paris, Calmann-Lévy, [1956] 1998 ; id., L'Enseignement du mépris, Paris [1960], Grasset, 2004.

${ }^{24}$ L. Poliakov, Histoire de l'Antisémitisme, 4 vol., Paris, Seuil, [1956-1977], 2 vol., 1984.

${ }^{25}$ B. Blumenkranz, parmi l'ensemble de son œuvre, Les Auteurs chrétiens latins du Moyen Âge sur les juifs et le judaïsme, [1963] Paris-Louvain, Peeters, 2007.
} 
l'un des savoirs sociaux les mieux partagés par les populations au-delà des chronologies temporelles et des géographies spatiales.

Il est notoire que le terme « antisémitisme » n'apparaît qu'au XIX ${ }^{\mathrm{e}}$ siècle sous la forme d'un positionnement politique ${ }^{26}$. Cependant, si l'appellation, entrée dans le langage courant, ne suffit guère à en rendre toutes les dimensions, ce qu'elle désigne est loin d'être défini clairement $^{27}$. Car, si on peut en situer l'émergence langagière, comment appréhender voire nommer ce qui l'a précédée? Cette interrogation incite à se demander, comme le fait Gavin Langmuir, si l'antijudaïsme ou la judéophobie - ne serait pas plutôt un phénomène aussi ancien que 1'existence des Juifs eux-mêmes ${ }^{28}$. Pour autant que l'anachronisme du terme et de son usage, typiquement ancré dans l'esprit de classification dix-neuviémiste des races, puisse être dépassé par une argumentation théorique à la Peter Schäfer et John Gager qui, tout en marquant leurs distances avec la simplification à outrance du terme reconnaissent la longévité de l'inimitié à l'égard des Juifs ${ }^{29}$, cette énigme se situe à la source de la tendance historiographique qui prévaut actuellement. Elle rejoint celle déjà défendue par Theodor Mommsen et Théodore Reinach en leur temps, d'un antisémitisme de toutes époques, irréductible à l'une ou l'autre de ses multiples facettes ${ }^{30}$.

Depuis le $\mathrm{XXI}^{\mathrm{e}}$ siècle, en effet, en débordant les barrières religieuses constituées par la chrétienté, ce savoir social, globalisé, s'est répandu de telle manière que les chercheurs se sont détournés des prémices posées par leurs prédécesseurs pour repenser en termes différents, et peut-être moins mesurés, l'hostilité ou la haine des Juifs,

\footnotetext{
${ }^{26}$ Parmi les innombrables travaux publiés sur ce thème, reste toujours utile le classique de J. Katz, From Prejudice to Destruction: Anti-semitism 1700-1933, Cambridge, Harvard University Press, 1980.

${ }^{27}$ Voir, par exemple, l'essai de sociologie de G. Erner, Expliquer l'antisémitisme, Paris, PUF, 2012.

${ }^{28}$ G. Langmuir, Toward a Definition of Antisemitism, Berkeley, University of California Press, 1996, Introduction, p. 4 sq; P. Schäfer, Judéophobie. Attitudes à l'égard des Juifs dans le monde antique, [1997] Paris, Cerf, 2003, ap. Theodore Mommsen qui situait synchroniquement diaspora et animosité à l'encontre des Juifs. Voir aussi sa discussion des conclusions de Langmuir dans le chap. "antisémitisme », Judeophobie..., op. cit., ainsi que sa «Response to Christine Hayes and Robert Goldenberg », Jewish Studies Quarterly, vol. 6, 1999, p. 274-281.

${ }^{29}$ P. Schäfer, Judeophobie..., op. cit. ; J. Gager, The Origins of Anti-Semitism. Attitudes toward Judaism in Pagan and Christian Antiquity, Oxford, University Press, 1985.

${ }^{30}$ T. Mommsen, Histoire romaine, vol. 5, Paris, Librairie Franck [Berlin 1884] 1866 ; T. Reinach, « Juif/Juifs, §Antisémitisme » La Grande Encyclopédie, Paris, Lamirault et cie, éditeurs, 1885-1902, t. 21, p. 277, à comparer cependant avec le ton mesuré qu'il emploie dans sa préface à Textes..., op. cit.
} 
désormais appréhendée comme une continuité quasi ininterrompue allant de l'Antiquité jusqu'à l'époque contemporaine. Cette orientation peut s'expliquer de mille manières, en partant des reconfigurations géopolitiques récentes jusqu'aux transformations des méthodes historiennes.

C'est surtout à ces dernières, pourtant, que cette mutation du champ historiographique, bien relevée par ailleurs, pourrait être attribuée, et pour laquelle les apports du travail sur la «longue durée» ont été les plus productifs. L'ouvrage de David Nirenberg Anti-Judaïsme ${ }^{31}$ en a donné le signal et à sa suite se sont engouffrées quantité d'études s'appuyant sur cette approche dont l'une, récente, appréhende cette attitude haineuse comme un élément intégré au rang des « archives » latentes de la mémoire collective, toujours prêt à resurgir en se parant de nouveaux atours pour peu que l'occasion s'y prête ${ }^{32}$.

En outre, l'introduction dans les pratiques de l'histoire d'analyses portant sur les émotions ou les images, pour ne mentionner que les plus fécondes, a permis d'exercer un autre regard sur le passé et donc d'en capturer différemment des pans entiers. On a ainsi tenté, en suivant Langmuir, d'élaborer des critères permettant de retrouver des continuités entre la haine " irrationnelle », médiévale, accompagnant les fantasmes les plus fantaisistes conduisant à des situations extrêmes, de la haine « rationnelle » accompagnant ses développements modernes, fondées sur des élucubrations racistes et/ou économiques et politiques. Si ces approches ont surtout entériné ce que l'on savait déjà par les sources textuelles concernant les attitudes à l'égard des Juifs dans le monde chrétien, on peut effectivement, en les saisissant au prisme de la longue durée, établir un fil conducteur qui permet, par exemple, de mettre en miroir certaines iconographies médiévales représentant des Juifs qui n'auraient rien à envier aux caricatures antisémites du XIX ${ }^{\mathrm{e}}$ siècle et dont il est possible de postuler une filiation ou une mémoire si ce n'est continue, tout au moins latente ${ }^{33}$.

\footnotetext{
${ }^{31}$ D. Nirenberg, Anti-Judaism : The Western Tradition, New York, W.W. Norton \& Company, 2013.

32 J. Adams et C. Heß (éd.), The Medieval Roots of Antisemitism. Continuities and Discontinuities from the Middle Ages to the Present Day, Londres, Routledge, 2018.

${ }^{33}$ Voir chez S. Lipton, Dark Mirror: The Medieval Origins of Anti-Jewish Iconography, New York, Henry Holt and Company, 2014, entre autres, chap. 5, la figure $\mathrm{n}^{\circ}$ 10, tirée du Psautier des Heures de Bonne de Luxembourg (1340), p. 191 ; ainsi que "What's in a Nose? The Origins, Development, and Influence of Medieval Anti-Jewish Caricature ", dans J. Adams et C. Heß, The Medieval Roots..., op. cit., p. $183-203$.
} 
Toutefois, ce n'est qu'en mettant à profit les sources issues de l'Antiquité gréco-romaine que cette approche a pu fonder sa cohérence. Or, c'est bien là que le bât blesse. Car, en dépit du fait pourtant bien attesté et régulièrement dénoncé par des cohortes d'hellénistes depuis les années $1980^{34}$, que la notion de « race » étant complétement étrangère à l'univers mental des Anciens, qui répartissait 1'humanité entre « grecs/ romains » et «barbares », et que l'on n'y pouvait déceler aucune trace d'idéologie à l'égard des Juifs ${ }^{35}$, cette approche semble actuellement s'imposer. On peut, certes, étayer les attitudes inamicales ou haineuses à l'encontre des Juifs par de multiples sources, textuelles ou iconographiques, allant du Moyen Âge jusqu'à l'époque contemporaine. Cela permet de déployer aisément des analyses fondées tant sur des événements que sur des recoupements textuels et iconographiques.

Il n'en va pas ainsi, tant s'en faut, pour ce qui concerne l'Antiquité. Car on le sait également, jusqu'à la transformation de l'Empire romain en empire chrétien et l'introduction au sein des textes juridiques de lois spécifiquement destinées aux Juifs, le corpus littéraire qui traite des Juifs, pour être répertorié et bien identifié par les collations effectuées par Théodore Reinach puis Menahem Stern ${ }^{36}$, n'en demeure pas moins assez succinct. Entièrement composé de vestiges textuels, le plus souvent hors contextes, et ayant plus ou moins bien franchi le barrage de siècles de transmissions par citations interposées, ce corpus ne permet de rendre compte ni des attitudes ni des sentiments répandus à leur égard. Car ces fragments comportent rarement des éléments permettant d'identifier leur provenance voire l'intention originelle de leurs auteurs. De fait, au delà

\footnotetext{
${ }^{34}$ L. Feldman, «Anti-Judaism, Josephus, and the Hellenistic-Roman Period », dans B. McGuire, A. Astell, et S. Goodhart (éd.), Sacrifice, Scripture, \& Substitution : Readings in Ancient Judaism and Christianity, Notre Dame, University Press, 2011, p. 208-226; S. Cohen "Anti-Semitism in 'Antiquity': The Problem of Definition », dans D. Berger, (éd.), History and Hate: The Dimensions of Anti-Semitism, Philadelphie/New York/Jérusalem, The Jewish Publication Society, 1986, p. 43-47; Z. Yavetz, "Judeophobia in Classical Antiquity. A different Approach », Journal of Jewish Studies, 44-1, 1993, p. 1-22 ; N. De Lange, "The Origins of Anti-Semitism: Ancient Evidence and Modern Interpretations », dans S. Gilman et S. Katz (éd.), AntiSemitism in Times of Crisis, New York, NYU Press, 1991, p. 21-37 ; etc.

${ }^{35}$ D. Nirenberg, op. cit., New York, W. W. Norton \& Company, 2013, c. 1 ; cf le dossier qui en a suivi la publication sur le forum Marginalia, http://marginalia.lareviewofbooks.org/judaism-hatred-vs-jew-hatred/; également, M. Kriegel, «L'esprit tue aussi. Juifs 'textuels' et Juifs 'réels' dans l'histoire », Annales HSS, 2014, p. 875-899.

${ }^{36}$ T. Reinach, Textes...,op. cit.; M. Stern, Greek and Latin Authors on Jews and Judaism, 3 vols., Jérusalem, The Israel Academy of Sciences and Humanities, 19741984.
} 
des périodes mouvementées du $\mathrm{I}^{\mathrm{er}}$ siècle de l'ère commune au cours duquel les habitants de la province de Judée se soulevèrent contre les Romains dans une guerre restée dans l'histoire grâce au stylet de Flavius Josèphe, peu d'auteurs anciens, grecs et romains, s'intéressèrent réellement à cette population pour le moins marginale.

Louis Feldman, qui a consacré ses travaux à l'histoire des Juifs dans l'Antiquité, s'était, dès 1988, fortement élevé contre les abus de la surinterprétation dans l'utilisation de textes des auteurs anciens. Pour montrer l'inanité de ces exercices, il avait ainsi recensé l'éventail des positions relevables au sein du corpus existant qui va de la plus lointaine antiquité au VII ${ }^{\mathrm{e}}$ siècle è.c, à l'exclusion des textes chrétiens qui en aurait forcément biaisé l'interprétation. En classant ainsi les mentions éparses de Juifs qui pourraient rendre compte des attitudes de leurs auteurs, qu'elles soient hostiles ou à l'inverse élogieuses, il a démontré que ce sont surtout les évocations «neutres» qui forment la majorité de la cohorte. Selon son analyse, cette large tranche chronologique comporte 111 témoignages amicaux ou bienveillants à l'égard des Juifs, soit 17\% du total ; les 140 fragments hostiles ou malveillants en représentent $22 \%$, tandis que 390 citations témoignent d'indifférence et forment ainsi une majorité de $61 \%$. Se prêtant au jeu de la statistique chronologiquement ventilée, Feldman a pu observer un léger mouvement de balancier durant la période de l'Empire (100 à 300) : ainsi a-t-il pu recenser que sur les 247 extraits mentionnant les Juifs au cours de ces deux siècles, 32 expriment de la sympathie, 40 révèlent de l'antipathie, et 176 ne dévoilent ni l'une ni l'autre. Plus encore, le passage au christianisme (300-600) ne marquerait, selon son recensement, pas même un changement particulièrement significatif, puisque 32 fragments présentent les Juifs avec avantage, 20 sont désobligeants, tandis que la majeure partie d'entre eux, soit 51 extraits, ne se prononcent en aucune manière ${ }^{37}$.

Or, si l'on dispose d'une connaissance approximative de la vie des Juifs et du judaïsme au $\mathrm{I}^{\mathrm{er}}$ siècle de l'ère commune grâce à deux informateurs, Philon d'Alexandrie et Flavius Josèphe, rien ne permet de se faire une idée de ce qu'a été le vécu juif au cours des siècles précédents hormis par l'intermédiaire de textes à valeur théologique comme le Nouveau Testament, les textes apocryphes ou encore les

\footnotetext{
${ }^{37}$ L. Feldman, « Pro-Jewish Intimations in Anti-Jewish Remarks Cited in Josephus' Against Apion », Jewish Quarterly Review, vol. 78, No. 3/4, jan. - apr. 1988, p. 187251.
} 
Rouleaux de Qumran et les rares mentions d'auteurs anciens. Acteurs et témoins, ces deux contemporains l'un de l'autre ont été préservés de l'oubli par l'intégration de leurs œuvres dans le patrimoine chrétien. Tandis que Philon témoigne de l'emprise hellénistique sur le judaïsme, il rapporte également l'un des événements anti-juifs parmi les plus marquants de l'Antiquité, dans le récit qu'il en fit en vue d'intercéder pour la communauté juive d'Alexandrie et obtenir l'appui et la protection de l'Empire. Toutefois, puisque c'est essentiellement à Josèphe que l'on doit la transmission de la plupart des anciens fragments traitant des Juifs, il est nécessaire de comprendre les raisons qui l'ont poussé à les recueillir et leur répondre, d'une part, et de l'autre, comment cette anthologie a pu traverser les siècles.

\section{Antisémitisme? Judéophobie? De quoi, et à qui, Josèphe parle-t-il ?}

Il faut donc revenir à Rome. Les diffamations contre les Juifs que Josèphe prétend combattre incarnent les stéréotypes les plus courants dans la culture hellénistique et ne semblent pas particulièrement réservés aux Juifs ${ }^{38}$. En ce sens, rien n'empêche de se demander si les calomnies que le Contre Apion a relevées étaient vraiment perçues pour infâmantes dans la société romaine ? Car en bon rhéteur, Josèphe les utilise à double tranchant. Qu'il s'agisse des supposées origines égyptiennes des Juifs tirées du récit du prêtre Manéthon, de celui du prêtre égyptien stoïque du $\mathrm{I}^{\mathrm{er}}$ siècle, Chærémon, et de Lysimaque dont on ne sait pas grand chose (ainsi que de celui d'Hécatée d'Abdère que Josèphe n'évoque pas) ${ }^{39}$, du

\footnotetext{
${ }^{38}$ Sur l'ensemble de ces questions la bibliographie abonde : B. Bar-Kochva, The Image of the Jews in Greek Literature. The Hellenistic Period, Berkeley, University of California Press, 2010 ; K. Berthelot, «The Use of Greek and Roman Stereotypes of the Egyptians by Hellenistic Jewish Apologists, with Special Reference to Josephus' Against Apion », dans J. Kalms, Internationales Josephus-Kolloquium Aarhus 1999, Münster, LIT, p. 185-221 ; E. Gruen, «Greek and Jews : Mutual Misperceptions in Josephus », dans C. Bakhos, Ancient Judaism and its Hellenistic Context, Ancient Judaism in its Hellenistic Context, Leyde, Brill, 2005, p. 31-51 ; J.-W. van Henten et R. Abusch, « The Jews as Typhonians and Josephus' Strategy of Refutation in Contra Apionem », dans L. Feldman et J. Levison, Josephus' Contra Apionem..., op. cit., p. 271-309.

39 Il faut probablement distinguer l'auteur grec d'une Histoire d'Égypte qui impute aux Juifs une origine égyptienne du Pseudo-Hécatée, auteur d'un "Sur les Judéens », souvent cité par Josèphe qui ne les distingue pas l'un de l'autre. Ce dernier serait, comme nombre d'autres auteurs, un Juif alexandrin écrivant sous un pseudonyme grec, voir B. Bar-Kochva, Pseudo Hecateus, "On the Jews»: Legitimizing the Jewish
} 
culte rendu à l'âne décrit dans la foulée de l'accusation de meurtre rituel (Ap. II 91-96), ou encore de leur haine à l'égard des étrangers, rapportées par Apion (Ap. II 121), ces charges prennent sous la plume de Josèphe une tournure polémique classique.

Pour ses contemporains, toutefois, les Égyptiens, perçus comme l'un des peuples les plus anciens et féru d'ésotérisme, étaient, quels qu'aient pu être les griefs ou la xénophobie à leur encontre, néanmoins hautement respectables ${ }^{40}$. Quant à l'exode des Juifs, situé selon les datations indiquées par Apion et rapportées par Josèphe ( $A p$. II 17), lors de la fondation de Carthage et de Rome, elles lui permettaient de se montrer simultanément au fait des méthodes et repères historiques grécoromains tout en ancrant l'histoire des Juifs dans la temporalité des origines du monde romain ${ }^{41}$.

Il en va de même des cultes animaux qui, pour être ridiculisés par les Romains, ne pouvaient être tenus pour abjects, puisque pratiqués par nombre de peuples dans l'ensemble du monde hellénistique. Les explications qu'il prête au mode de vie des Juifs s'inspirent de celui des Spartiates, hautement valorisé à cette époque ${ }^{42}$. De fait, si Josèphe dresse un inventaire des critiques anti-juives circulant dans le monde grec (d'où l'appellation de son livret par Porphyre «Contre les Grecs » ${ }^{43}$ ) c'est pour mieux les retourner contre leurs auteurs et monter une apologie des Juifs et du judaïsme en bonne et due forme ${ }^{44}$. Ses réfutations portent tant sur les railleries du shabbat dues au géographe Agartharchide de Cnide

Diaspora, Berkeley, California Press, 1996 ; T. Reinach, Textes..., op. cit., p. 14- 20 ; 14-15 ; M. Stern, Greek and Latin Authors..., op. cit., 1976, vol 1, p. 22-24 ; E. Schürer, The History of the Jewish People in the Age of Jesus Christ (175 B.C.-A.D. 135). Revised and edited by G. Vermes and F. Millar, Edinburgh, T. \& T. Clark, 1973, III. p. 672-673. La description du débat scientifique : J. Barclay, « Appendix 2 : PseudoHecateus », dans S. Mason (éd.), Flavius Josephus... op. cit., p. 338-340.

${ }^{40} \mathrm{~B}$. Isaac, The Invention of Racism in Classical Antiquity, Princeton, Oxford University Press, 2004, chap. 7.

${ }^{41}$ L. Feldman, "Reading... ", op. cit., p. 268. D. Potter, "The Greek Historians of Imperial Rome », dans A. Feldherr et G. Hardy (éd.) The Oxford Dictionary of Historical Writings, vol. 1, Beginnings to AD 600, Oxford, Oxford University Press, 2011, p. 316- 345. G. Bowersock, Augustus and the Greek World, Oxford, Clarendon Press, [1965] 1996.

${ }^{42}$ L. Feldman, « Reading... », op. cit., p. 254 ; K. Berthelot, Philanthrôpia judaica. Le débat autour de la 'misanthropie' des lois juives dans l'Antiquité, Leyde, Brill, 2003, p. 362-366.

${ }^{43}$ Porphyre, De Abstinentia, IV 11.

${ }^{44}$ J. Barclay, « The Politics of Contempt : Judeans and Egyptians in Josephus' Against Apion », dans id., Negotiating Diaspora: Jewish Strategies in the Roman Empire, Londres, T\&T Clark International, 2004, p. 109-127. 
(II ${ }^{\mathrm{e}}$ siècle av. è.c.) que sur le récit inversé de la sortie d'Égypte, circulant apparemment dans certains cercles, qui décrit l'expulsion des Juifs d'Égypte pour cause de dissémination de diverses maladies infầmes, dont la lèpre (Lysimaque), ou encore leur refus d'honorer les statues impériales, et nombre d'autres fables parfois saugrenues rapportées par sa cible favorite : Apion. Ce dernier, à en croire Josèphe, aurait récupéré l'ensemble des attaques éparpillées chez divers auteurs, y compris les critiques de la circoncision et de la prohibition du porc, auxquelles il aurait ajouté quelques fantaisies de son cru. Ce faisant, tournant en dérision les «Égyptiens » et leur culte, cible convenue à son époque, Josèphe rehausse bel et bien l'image du judaïsme qu'il présente sous son jour le plus propice : entre philosophie et art de vivre selon les critères les plus hautement valorisés en son temps.

Au-delà de la réfutation de ces calomnies, c'est l'apologie qui domine le sens du texte : Josèphe, en pourfendant les Grecs pour leur futilité et leur récente apparition dans l'histoire, s'affirme en loyal citoyen romain. En outre, c'est par l'intermédiaire des anciennes mentions de Juifs qu'il entend démontrer l'antiquité de son peuple (et donc sa valeur historique), de sorte que tout en faisant mine de défendre le judaïsme contre des attaques injustifiées, il attire, surtout, l'attention de son lectorat d'auditeurs sur les valeurs et la suprématie du judaïsme ${ }^{45}$. S'appuyant sur des extraits cités hors contexte, il tisse un patchwork, peut-être convaincant pour ses lecteurs, de la grandeur et de la rectitude du judaïsme et de l'histoire des Juifs et n'hésite ni à omettre les passages qui ne privilégieraient pas son argumentation ni à transformer en diffamations aisément réfutables des textes qui, à l'origine, ne s'intéressaient aux Juifs qu'en passant et ne comportaient peut-être pas la moindre connotation péjorative.

C'est que Josèphe écrivait pour ses contemporains, qu'ils soient juifs ou païens. S'il est malheureusement vain d'imaginer les réactions de son auditoire à cette apologie, la postérité n'en ayant pas gardé trace, on peut néanmoins suivre celle des lectures et des interprétations qui en ont été faites au cours des siècles suivants. Paradoxalement, les allégations que Josèphe avait combattues et ridiculisées seront, justement, celles qui s'inscriront le plus profondément dans les mémoires.

\footnotetext{
${ }^{45}$ M. Goodman, Mission and Conversion. Proselytizing in the Religious History of Roman Empire, Oxford, Clarendon Press, [1994] 1996; S. Mason, " The Contra Apionem in Social and Literary Context : An Invitation to Judean Philosophy », dans L. Feldman et J. Levison, Josephus' Contra Apionem..., op. cit., p. 187- 228.
} 
Josèphe fut en effet utilisé dès les deux premiers siècles de l'ère dans les sermons chrétiens qui y puisèrent sans scrupule de quoi alimenter la théologie de la substitution. Si Méliton, évêque de Sarde, et Hyppolyte de Rome ( $c a$ 170-235) furent les premiers à s'en servir, c'est Origène ( $c a$ 185-253) qui en tira l'interprétation anti-juive de la chute de Jérusalem, directement détournée de passages des Antiquités : ainsi la destruction du Temple était-elle venue châtier le crime des Juifs à l'encontre de Jacques et leur rejet de Jésus ( $A J$ XVIII 118-119, XX 200, XVIII 63-64). L'argument fut amplement complété par le génial propagandiste Eusèbe, évêque de Césarée ( ca 260-339/340), qui désigna le sort fatal de Jérusalem comme la preuve éclatante et historique de la vérité du christianisme. Sans hésiter à falsifier l'interprétation de Josèphe, qui décrivait l'ampleur du nombre de combattants déportés et vendus en tant qu'esclaves après la chute de la Judée, Eusèbe déclara sans ambages que la tardive invention chrétienne de la «servitude » des Juifs, et donc leur dispersion, découlait de leur crime (n'avoir pas reconnu le Sauveur en Jésus), et avait démarré dès la destruction du Temple en $70^{46}$. Et ce en dépit de la présence continue et persistante des Juifs en Palestine au cours des siècles suivants - qu'il ne pouvait ignorer au sein de son propre environnement ${ }^{47}$ - et du fait que le judaïsme avait maintenu son statut légal dans tout l'Empire romain au cours des siècles suivant les deux insurrections ${ }^{48}$. Plus encore, pour mieux faire coïncider les signes célestes avec les événements historiques, Eusèbe n'hésita pas à transférer la chute du Temple lors de la fête de Pâque - au printemps - au lieu du mois de $A v$ - en été - afin qu'elle corresponde avec la date de la crucifixion $^{49}$.

\footnotetext{
${ }^{46}$ R. Zachman, «Identity, Theology and the Jews: The Uses of Jewish Exile in the Creation of Christian Identity », dans E. O’Donnell Polyakov (éd.), Antisemitism, Islamophobia, and Interreligious Hermeneutics. Ways of Seeing the Religious Others, Leyde, Brill, 2019, p. 51-67.

${ }^{47}$ D. Goodblatt, "The Political and Social History of the Jewish Community in the Land of Israel, c. 235-638», dans S. Katz (éd.), The Cambridge History of Judaism, vol. IV, The Late Roman-Rabbinic Period, Cambridge, Cambridge University Press, 2006, p. 404-430, 406.

${ }^{48}$ La situation des Juifs ne se serait dégradée dans l'Empire qu'après son passage au christianisme, à la fin du $\mathrm{IV}^{\mathrm{e}}$ siècle et au long du V $\mathrm{V}^{\mathrm{e}}$ siècle. E. Smallwood, The Jews under Roman Rule. From Pompey to Diocletian, Leyde, Brill, 1976, p. 539-542; J. Juster, Les Juifs dans l'empire romain. Leur condition économique et sociale, t. 2, Paris, Paul Geuthner, 1914, p. 322-326.

${ }^{49}$ Histoire Ecclésiastique, III. 5-12, et VI, 425-428 ; H. Schreckenberg, " The Work of Josephus », dans L. Feldman et G. Hata (éd.), Josephus, Judaism and Christianity, Detroit, Wayne State University Press, 1987, p. 320.
} 
Le Juif Josèphe pénétra l'héritage littéraire chrétien, au prix de sérieuses distorsions, sous la plume de Jérôme (347-420). Il l'inclut dans son Histoire de la littérature chrétienne (De Viris Illustribus), avec un chapitre se présentant comme un substitut de la Vita qui, apparemment, ne fut pas autrement traduite en latin durant l'Antiquité. De même, le Pseudo-Hegesippus ( ca 370), une édition latine du IV ${ }^{\mathrm{e}}$ siècle $^{50}$, altérera la version grecque de Guerre en l'intégrant dans la polémique anti-juive ${ }^{51}$. Tout en louant le témoignage en faveur de Jésus apporté (probablement via interpolation) par Josèphe, le texte lui reproche la dureté de son cœur (duritia cordis) et son refus obstiné (perfidia) de reconnaître le Sauveur en la personne de Jésus ${ }^{52}$.

L'incorporation de Josèphe au cœur de l'histoire chrétienne fut si phénoménale que le moine anglais Bède le Vénérable (ca 673-735), le classa parmi les Pères de l'Église, dans la lignée d'Origène, Jérôme et Augustin $^{53}$. Cette récupération illustre le combat chrétien mené à partir du judaïsme pour mieux le récuser. Déviant le texte de la Guerre, transférant sa condamnation de l'entêtement des zélotes à refuser de rendre les armes vers l'argument théologique de l'obstination des Juifs, Josèphe fut systématiquement utilisé à leur encontre et fournit même la pièce maîtresse de leurs chefs d'accusation. Plus encore, pour convertir la victoire romaine en victoire chrétienne, la Guerre de Josèphe fut placée en gage de témoignage irréfutable en appendice à nombre d'éditions imprimées du Nouveau Testament et de livres de prières, et la coutume fut même instaurée d'en lire des extraits significatifs au dixième dimanche suivant la Trinité ${ }^{54}$. Et que la Guerre de Josèphe rédigée originellement en araméen (sans qu'il en reste trace), puis traduite en grec, ait connue sa plus grande gloire dans sa version latine, atteste les voies de sa pérennité.

\footnotetext{
${ }^{50}$ Sur les éditions latines de l'œuvre de Josèphe: D. Levenson et T. Martin, «The Ancient Latin Translations of Josephus », dans H.H. Chapman et Z. Rodgers (éd.), $A$ Companion..., op. cit., p. 322-344.

${ }^{51}$ A. Wealey, «The Testimonium Flavianum » et l'abondante bibliographie y afférant dans H.H. Chapman et Z. Rodgers (éd.), A Companion..., op. cit., p. 345-355.

52 Corpus Scriptorum Ecclesiasticorum Latinorum, 66.1.3 et 66.1 .164 ; H. Schreckenberg, « The Work... », art. cit., 1987, p. 315-324.

${ }^{53}$ Patrologia Latina, éd. Migne, 94, 675.

${ }^{54}$ H. Schreckenberg, "The Work... », art. cit., 1987, p. 319 ; H. Schreckenberg et K. Schubert, Historiography and iconography in Early and Medieval Christianity, Aassen/Maastricht, Von Gorkum, Minneapolis Fortress Press, 1992, p. 10.
} 
Quant au catalogue collecté par Josèphe dans le Contre Apion, habilement détourné, inséré dans le répertoire chrétien des invectives usuelles, il alimentera les formes consacrées des accusations antijuives ${ }^{55}$. Et on peut certes discerner dans ce recueil de charges l'arrière-fond d'antiennes chrétiennes ignominieuses qui circuleront sur les Juifs à partir de l'Antiquité tardive : l'origine égyptienne du peuple juif peut renvoyer au statut d'éternel étranger, le culte de l'âne - visant à ridiculiser son monothéisme - se muer en pratiques infâmes et enfin, bien que plus étrange à l'origine, l'imputation de pratiquer le meurtre rituel renvoyant aux sacrifices païens - a pu connaître au long des siècles un succès durable ${ }^{56}$. D'autres encore, que Josèphe a patiemment assemblées, telles que la misanthropie ${ }^{57}$ ou l'athéisme/impiété, accusations généreusement partagées dans l'Antiquité avec nombre d'autres peuples considérés comme barbares ${ }^{58}$, deviendront l'apanage exclusif des Juifs durant le Moyen Âge.

Et, loin de se contenter de puiser dans l'œuvre de Josèphe des sources accommodables à ses desseins diffamatoires, l'Église aura également su tirer parti de la propagande romaine liée à la défaite de la Judée : l'iconographie de la monnaie romaine Iudea capta montrant une femme en pleurs près d'un palmier, peut se retrouver dans la célèbre allégorie de la synagogue aux yeux bandés ${ }^{59}$. Mais qui saurait dire par quel étrange canal les sculpteurs des cathédrales au Moyen Âge et les caricaturistes du XIX ${ }^{\mathrm{e}}$ siècle ont-ils pu exhumer des images qu'ils n'avaient probablement jamais vues ? S'agit-il de ces matériaux inertes que la mémoire collective aurait intégrés et fait ressurgir après une éclipse de plusieurs siècles?

\footnotetext{
${ }^{55}$ Dans l'immense littérature traitant de l'antijudaïsme en sus des auteurs déjà cités, voir l'« Épilogue» dans M. Goodman, Rome et Jérusalem..., op. cit.; K. Stow, Jewish Dog's. An Image and its Interpreters, Stanford, University Press, 2006 ; R. Chazan, From Anti-Judaism to Anti-Semitism. Ancient and Medieval Christian Constructions of Jewish History, New York, Cambridge University Press, 2016.

${ }^{56} \mathrm{~J}$. Tokarska-Bakir, Les Légendes du sang: Une anthropologie du préjugé antisémite en Pologne (1600-2005), Paris, Albin Michel, 2015.

${ }^{57}$ K. Berthelot, Philanthrôpia ..., op. cit., p. 106-143.

${ }^{58}$ B. Isaac, The Invention..., op. cit., 2004 ; G. Bohak, « The Ibis and the Jewish Question: Ancient 'Anti-semitism' in Historical Perspective», dans M. Mor, A. Oppenheimer et al., (éd.), Jews and Gentiles Relations in the Holy Land in the Days of the Second Temple, The Mishnah and the Talmud, Jérusalem, Yad Ben-Zvi, 2003, p. $27-43$.

${ }^{59}$ E. Revel-Neher, «An 'Encore' on the Bar Kochba Tetradrachm : A Re-vision of Interpretation ", dans Z. Weiss et al. (éd.), Follow the Wise : Studies in Jewish History and Culture in Honor of Lee I. Levine, Winona Lake, Eisenbrauns, 2010, p. 189-206.
} 


\section{Temps et histoire : l'arsenal du chrétien}

Celui-ci a eu, par hasard, pour père un observateur du sabbat : il n'adorera que les nuages et la divinité du ciel; il ne fera aucune différence entre la chair humaine et celle du porc, dont s'est abstenu son père; bientôt même il se fait circoncire. Élevé dans le mépris des lois romaines, il n'apprend, n'observe, ne révère que la loi judaïque, tout ce que Moïse a transmis à ses adeptes dans un volume mystérieux : ne pas montrer la route au voyageur qui ne pratique point les mêmes cérémonies ; n'indiquer une fontaine qu'au seul circoncis. Et tout cela parce que son père passa dans l'inaction chaque septième jour, sans prendre aucune part aux devoirs de la vie. (Juvénal $\mathrm{I}^{\mathrm{er}}-\mathrm{II}^{\mathrm{e}}$ (?) siècles). ${ }^{60}$

Dans la Rome des premiers siècles, on le sait, l'attirance vers le monothéisme était puissante et la concurrence entre juifs et chrétiens, rude. C'est d'ailleurs le phénomène important des conversions vers le judaïsme qui devrait alerter quant à la réalité d'une atmosphère haineuse répandue dans l'Empire romain. Et ce d'autant, que si le culte juif était tenu pour légitime, celui des chrétiens ne jouissait pas des mêmes privilèges $^{61}$. L'histoire de la transmission des écrits de Flavius Josèphe permet ainsi de saisir de manière exemplaire comment les chrétiens ont développé et édifié leur histoire sainte ${ }^{62}$. Car on le sait également, en offrant une reconstruction temporelle et historique de l'univers, ce récit permettait au converti récent d'intégrer un monde divinement orienté qui insérait son présent dans une histoire ébauchée à l'aube des temps et déterminée par sa fin ${ }^{63}$.

En s'appropriant un passé et une histoire auxquels ils n'avaient pas été confrontés, les chrétiens se font légataires d'un message qui devait, cependant, être transformé et amplifié. Pour les apologues et les évangélistes des premiers siècles, la tâche était délicate : ils devaient à la fois magnifier les valeurs du judaïsme prônées dans la Bible et transmises par les Juifs tout en se différenciant autant que faire se peut de ces derniers.

En ce sens, le Contre Apion rencontra les attentes des apologistes chrétiens qui devaient simultanément se défendre contre des accusations

\footnotetext{
${ }^{60}$ Juvénal, Satire 172 dans T. Reinach, Textes..., op. cit., p. 292-293.

${ }^{61}$ Sur le lent passage au christianisme, voir notamment P. Brown, À travers un trou d'aiguille. La richesse, la chute de Rome et la formation du christianisme, Paris, Les Belles-Lettres, 2016.

${ }^{62}$ K. Kletter, "The Christian Reception of Josephus in Late Antiquity and the Middle Ages », dans H.H. Chapman et Z. Rodgers (éd.), A Companion ..., op. cit., p. 368-378.

${ }^{63}$ P. Ariès, Le temps de l'histoire, Paris, Seuil, [1954] 1986, p. 96.
} 
d'infamie et assurer leur position historico-théologique dans le monde romain. Procurant le savoir historique comme la connaissance théologique, tout en présentant un modèle d'apologie classique, l'ouvrage occupa une place providentielle dans les constructions des Pères de l'Église : le texte leur permettait de reprendre à leur compte non seulement sa démonstration de la noblesse et de l'ancienneté du monothéisme et de l'éthique biblique, surpassant en cela toutes les autres croyances, mais également de muer la géographie biblique en géographie de la Terre sainte ${ }^{64}$; tout en se servant des accusations dont Josèphe défendait les Juifs pour en conclure qu'ils avaient suscités la répulsion et la haine depuis la plus lointaine antiquité. De sorte que cette conversion du «savoir» juif en «savoir» chrétien offrait un atout indéniable, puisqu'elle permettait, en affublant les Juifs des pires iniquités, d'en faire le repoussoir naturel de tout bon chrétien.

On pourrait effectivement situer là l'émergence de la figure du Juif «herméneutique/textuel» qui est chère aux approches récentes de l'historiographie $^{65}$. Ce n'est pourtant qu'avec la localisation dans le temps et l'espace des diatribes les plus percutantes contre les Juifs, telles que celles proférées par Jean Chrysostome, Jérôme ou encore Eusèbe qui avaient fréquentés et disputés avec de "vrais» Juifs, que ce soit à Antioche, Césarée et Rome, que s'éveille le doute quant à la justesse de l'analyse du moins pour ce qui a trait à l'Antiquité tardive. Et ce, d'autant plus qu'en considérant la menace exercée par l'attirance des gentils vers le judaïsme, attestée par les sources romaines, on saisit peutêtre la raison pour laquelle ce sont les passages qui devaient paraitre les plus laudatifs à un auditoire/lectorat païen à l'égard du judaïsme qui manquent dans les manuscrits grecs du Contre Apion ${ }^{66}$.

Il me faut préciser, en guise de conclusion, que cette contribution n'entendait ni apporter une réponse ni prendre position au sujet de la manière théorique dont il faudrait ou non appréhender l'hostilité, l'animosité voire la haine à l'égard des Juifs au long et à travers les âges. Il s'agissait, plus modestement, en adepte assidue du regard historique de longue durée, d'en éprouver la validité systématique en exposant un cas

\footnotetext{
${ }^{64}$ A. Jacob, The Remains of the Jews. The Holy Land and Christian Empire in Late Antiquity, Stanford, University Press, 2004, p. 34 ; Y. Shahar, Josephus Geographicus : The Classical Context of Geography in Josephus, Tübingen, Mohr Siebeck, 2004.

${ }^{65}$ M. Kriegel, « L'esprit tue aussi... ». art. cit.

${ }^{66}$ L. Feldman, « Reading... », op. cit., p. 250-252.
} 
d'étude exemplaire tout en ajoutant, si tant est que cela soit encore possible, de la complexité à un dossier déjà lourdement chargé.

$* * * * * * * * * * * * * *$

\section{Bibliographie}

Adams Jonathan et Heß Cordelia (éd.), The Medieval Roots of Antisemitism. Continuities and Discontinuities from the Middle Ages to the Present Day, New York/Londres, Routledge, 2018.

Ariès Philippe, Le temps de l'histoire, Paris, Seuil, [1954] 1986.

Bar-Kochva Bezalel, Pseudo Hecateus, "On the Jews" : Legitimizing the Jewish Diaspora, Berkeley, California Press, 1996.

Bar-Kochva Betzalel, The Image of the Jews in Greek Literature. The Hellenistic Period, Berkeley, California Press, 2010.

Barclay John M. G., Negotiating Diaspora: Jewish Strategies in the Roman Empire, Londres, T\&T Clark International, 2004.

Barclay John M. G., Flavius Josephus : Translation and commentary, éd. Steve Mason, vol. 10, Against Apion, Leyde/Boston, Brill, 2007.

Berthelot Katell, «The Use of Greek and Roman Stereotypes of the Egyptians by Hellenistic Jewish Apologists, with Special Reference to Josephus' Against Apion », dans J.U. Kalms, Internationales JosephusKolloquium Aarhus 1999, Münster, LIT, p. 185-221.

Berthelot Katell, Philanthrôpia judaica. Le débat autour de la 'misanthropie' des lois juives dans l'Antiquité, Leyde, Brill, 2003.

Blumenkranz Bernhard, Les Auteurs chrétiens latins du Moyen Âge sur les juifs et le judaïsme, [1963] Paris-Louvain, Peeters, 2007.

Bohak Gideon, "The Ibis and the Jewish Question: Ancient 'Antisemitism' in Historical Perspective », dans M. Mor, A. Oppenheimer et al., Jews and Gentiles Relations in the Holy Land in the Days of the Second Temple, The Mishnah and the Talmud, Jérusalem, Yad Ben-Zvi, 2003, p. 27-43. 
Bowersock Glen W., Augustus and the Greek World, Oxford, Oxford at Clarendon Press, [1965] 1996.

Brown Peter, À travers un trou d'aiguille. La richesse, la chute de Rome et la formation du christianisme, Paris, Les Belles-Lettres, 2016.

Chapman Honora Howell et Rodgers Zuleika (éd.), A Companion to Josephus, Oxford, Wiley-Blackwell, 2016.

Chazan Robert, From Anti-Judaism to Anti-Semitism. Ancient and Medieval Christian Constructions of Jewish History, New York, Cambridge University Press, 2016.

Cohen Shaye J. D., "Anti-Semitism in 'Antiquity': The Problem of Definition », dans D. Berger, (éd.), History and Hate: The Dimensions of Anti-Semitism, Philadelphie/New York/Jérusalem, The Jewish Publication Society, 1986, p. 43-47.

De Lange Nicholas, «The Origins of Anti-Semitism : Ancient Evidence and Modern Interpretations ", dans S. L. Gilman et S. T. Katz (éd.), Anti-Semitism in Times of Crisis, New York, NYU Press, 1991, p. 2137.

Dillery John, «Putting Him Back Together Again: Apion Historian, Apion Grammatikos », Classical Philology, 98, 4, Octobre 2003, p. 383-390.

Erner Guillaume, Expliquer l'antisémitisme, Paris, PUF, 2012.

Feldman Louis H., "Pro-Jewish Intimations in Anti-Jewish Remarks Cited dans Josephus' "Against Apion" ", Jewish Quarterly Review, New Series, Vol. 78, No. 3/4, Jan -Apr., 1988, p. 187-251.

Feldman Louis H., "Reading Between the Lines: Appreciation of Judaism in Anti-Jewish Writers Cited in Contra Apionem », dans L. Feldman et J. Levison (éd.), Josephus' Contra Apionem, 1996, p. 250270.

Feldman Louis H., Josephus' Interpretation of the Bible, Berkeley, University of California Press, 1998.

Feldman Louis H., «Financing the Colosseum », Biblical Archaeology Review 27, 4, Juillet-Août 2001, p. 20-31 et p. 60 - 61.

Feldman Louis H., " Anti-Judaism, Josephus, and the Hellenistic-Roman Period », dans B.P. McGuire, A.W. Astell, et S. Goodhart (éd.), Sacrifice, Scripture, \& Substitution : Readings in Ancient Judaism and Christianity, Notre Dame, Notre Dame University Press, 2011, p. 208226.

Feldman Louis H. et Hata Gohei, Josephus, Judaism and Christianity, Detroit, Wayne State University Press, 1987. 
Feldman Louis H. et Levison John (éd.), Josephus' Contra Apionem : Studies in its Character and Context with a Latin Concordance to the Portion Missing in Greek, Leyde, Brill, 1996.

Gager John G., The Origins of Anti-Semitism. Attitudes toward Judaism in Pagan and Christian Antiquity, New York/ Oxford, Oxford University Press, 1985.

Goldberg Sylvie Anne, "L'Amère ironie de l'histoire », dans Flavius Josèphe. Contre Apion, Paris, Les Belles Lettres, 2018, p. VI-XCII.

Goodblatt David, "The Political and Social History of the Jewish Community in the Land of Israel, c. 235-638», dans S. Katz (éd.), The Cambridge History of Judaism, vol. IV, The Late Roman-Rabbinic Period, Cambridge, Cambridge University Press, 2006, p. 404-430.

Goodman Martin, The Ruling Class of Judea : The Origins of the Jewish Revolt Against Rome A.D. 66-70, Cambridge /New York, Cambridge University Press [1987] 1989.

Goodman Martin, «Josephus as a Roman Citizen», dans F. Parente et J.Sievers, Josephus and the History of the Greco-Roman Period. Essays in Memory of Morton Smith, Leyde, Brill, 1994, p. 329-338.

Goodman Martin, Mission and Conversion. Proselytizing in the Religious History of Roman Empire, Oxford, Clarendon Press, [1994] 1996.

Goodman Martin, Rome et Jérusalem. Le choc des civilisations, Paris, Perrin [2009] 2011.

Gruen Erich S., « Greek and Jews : Mutual Misperceptions in Josephus », dans C. Bakhos, Ancient Judaism and its Hellenistic Context, Leyde/Boston, Brill, 2005, p. 31-51.

Halporn James W., Cassiodorus : Institutions of Divine and Secular Learning and on the Soul, Liverpool, Liverpool University Press, 2003.

Isaac Benjamin, The Invention of Racism in Classical Antiquity, Princeton, N. J. Oxford, Oxford University Press, 2004.

Isaac Jules, Genèse de l'antisémitisme. Essai historique, Paris, CalmannLévy, [1956] 1998.

Isaac Jules, L'Enseignement du mépris, Paris [1960], Grasset, 2004.

Jacob Andrew S., The Remains of the Jews. The Holy Land and Christian Empire in Late Antiquity, Stanford, Stanford University Press, 2004.

Jones Brian W., The Emperor Domitian, New York, Routledge, 1992.

Katz Jacob, From Prejudice to Destruction : Anti-semitism 1700-1933, Cambridge, Harvard University Press, 1980.

Kriegel Maurice, «L'esprit tue aussi. Juifs 'textuels' et Juifs 'réels' dans l'histoire », Annales HSS, 2014, p. 875-899. 
Langmuir Gavin, Toward a Definition of Antisemitism, Berkeley, University of California Press, 1996.

Lipton Sara, "What's in a Nose? The Origins, Development, and Influence of Medieval Anti-Jewish Caricature », dans J. Adams et C. $\mathrm{He} \beta$, The Medieval Roots of Antisemitism, Continuities and Discontinuities from the Middle Ages to the Present Day, New York/Londres, Routledge, 2018, p. 183-203.

Lipton Sara, Dark Mirror: The Medieval Origins of Anti-Jewish Iconography, New York, Henry Holt and Company, 2014.

Marginalia Forum, http://marginalia.lareviewofbooks.org/judaismhatred-vs-jew-hatred/

Mark Edwards, Goodman Martin et Price Simon, avec Rowland Christopher (éd.), Apologetics in the Roman Empire: Pagans, Jews, and Christians, Oxford, Oxford University Press [1999] 2002.

Mason Steve, «The Contra Apionem in Social and Literary Context : An Invitation to Judean Philosophy ", dans L. Feldman et J. Levison, Josephus' Contra Apionem: Studies in its Character and Context with a Latin Concordance to the Portion Missing in Greek, Leyde, Brill, 1996, p. 187- 228.

Mason Steve, "Flavius Josephus in Flavian Rome: Reading On and Between the Lines ", dans A.J. Boyle et W.J. Dominik (éd.), Flavian Rome : Culture, image, text, Leyde-Boston, Brill, 2003, p. 559-590.

Mason Steve, A History of the Jewish War A.D. 66-70, Cambridge, Cambridge University Press, 2016.

Moscati Mascetti Giordana, The 'Hidden Transcripts' in Against ApionJosephus' Silent Criticism of Flavian Rome, PHD Thesis, Bar-Ilan University, Bar Ilan, 2011.

Nirenberg David, Anti-Judaism: The Western Tradition, New York, W. W. Norton \& Company, 2013.

Parkes James, The Conflict of the Church and the Synagogue : A Study in the Origins of Anti-Semitism, Londres, Soncino Press, 1934.

Poliakov Léon, Histoire de l'Antisémitisme, 4 vol., Paris, Seuil, [19561977] 2 vol., 1984.

Potter David, "The Greek historians of imperial Rome", dans A. Feldherr et G. Hardy (éd.) The Oxford Dictionary of Historical Writings, vol. 1, Beginnings to AD 600, Oxford, Oxford University Press, 2011, p. 316- 345.

Reinach Théodore, s.c. «Juif/Juifs, § Antisémitisme», La Grande Encyclopédie, Paris, H. Lamirault et cie, éditeurs, 1885-1902, t. 21. 
Reinach Théodore, Textes d'auteurs grecs et romains relatif au Judaïsme, Hildesheim/Zurich/ New York, Georg Olms, [1895] 1963.

Revel-Neher Elisheva, "An 'Encore' on the Bar Kochba Tetradrachm : A Re-vision of Interpretation », dans Z. Weiss et al. (éd.), Follow the Wise : Studies in Jewish History and Culture in Honor of Lee I. Levine, Winona Lake, Indiana, Eisenbrauns Inc., 2010, p. 189-206.

Schäfer Peter, "Response to Christine Hayes and Robert Goldenberg », Jewish Studies Quarterly, Vol. 6, 1999, p. 274-281.

Schäfer Peter, Judéophobie. Attitudes à l'égard des Juifs dans le monde antique, [Harvard University Press 1997] Paris, Cerf, 2003.

Schrekenberg Heinz, Die Flavius-Josephus-Tradition in Antike und Mittelalter, Leyde, Brill, 1972.

Schreckenberg Heinz, Rezeptionsgeschichtliche und Textkritische Untersuchungen zu Flavius Josephus, Leyde, Brill, 1977.

Schreckenberg Heinz, "The Work of Josephus », dans L. Feldman et G. Hata (éd.), Josephus, Judaism and Christianity, Detroit, Wayne State University Press, 1987, p. 315- 324.

Schreckenberg Heinz et Schubert Kurt, Historiography and Iconography in Early and Medieval Christianity, Aassen-Maastricht, Von Gorkum, Minneapolis Fortress Press, 1992.

Shahar Yuval, Josephus Geographicus: The Classical Context of Geography in Josephus, Tübingen, Mohr Siebeck, 2004.

Smallwood E. Mary, The Jews under Roman Rule. From Pompey to Diocletian, Leyde, Brill, 1976.

Stern Menahem, Greek and Latin Authors on Jews and Judaism, 3 vols., Jérusalem, The Israel Academy of Sciences and Humanities, 19741984.

Stow Kenneth, Jewish Dog's. An image and its Interpreters, Stanford, Stanford University Press, 2006.

Tokarska-Bakir Joanna, Les Légendes du sang: Une anthropologie du préjugé antisémite en Pologne (1600-2005), Paris, Albin Michel, 2015.

Tomson Peter J., Schwartz Joshua (éd.), Jews and Christians in the First and Second Centuries : How to Write their History, Leyde, Brill, 2014.

Trachtenberg Joshua, The Devil and the Jews : The Medieval Conception of the Jew and its Relation to Modern Antisemitism, [1943] Philadelphie, JPS, 2002.

Van der Horst Pieter Willem, «Who Was Apion?» dans id. (éd.), Japheth in the Tents of Shem: Studies on Jewish Hellenism in Antiquity, Leuven, Peeters, 2002, p. 207-221. 
Van der Horst Pieter Willem, Philo's Flaccus: The First Pogrom: Introduction, Translation, and Commentary, Leyde, Brill, 2003.

Van Henten Jan-Willem et Abusch Ra'anan, «The Jews as Typhonians and Josephus' Strategy of Refutation in Contra Apionem » dans L. Feldman et J. Levison, Josephus' Contra Apionem: Studies in its Character and Context with a Latin Concordance to the Portion Missing in Greek, Leyde, Brill, 1996, p. 271-309.

Wilson Marcus, "After the Silence : Tacitus, Suetonius, Juvenal », dans A.J. Boyle et W.J. Dominik (éd.), Flavian Rome : Culture, Image, Text, Leyde-Boston, Brill, 2003, p. 523-542.

Yavetz Zvi, «Judeophobia in Classical Antiquity. A Different Approach », Journal of Jewish Studies, 44-1, 1993, p. 1-22.

Zachman Randall C., "Identity, Theology and the Jews: The Uses of Jewish Exile in the Creation of Christian Identity », dans E. O'Donnell Polyakov (éd.), Antisemitism, Islamophobia, and Interreligious Hermeneutics. Ways of Seeing the Religious Others, Leyde/ Boston, Brill, 2019, p. 51-67. 
Les œuvres de Flavius Josèphe avec trois dissertations par William Whiston, édition londonienne de 1825

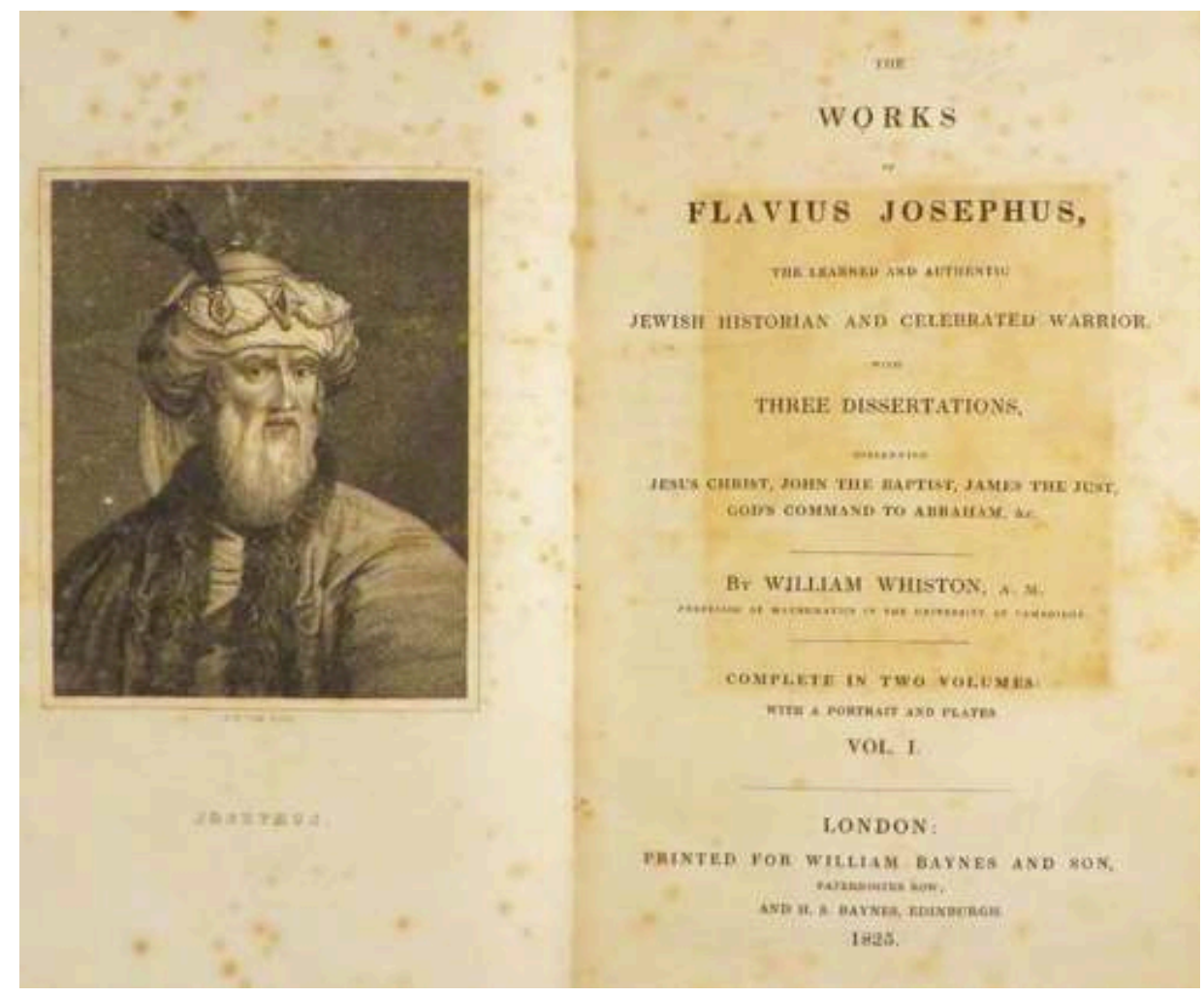

